\title{
La Historia Enseñada: una mirada los cambios en las permanencias
}

\author{
Maria Paula Gonzalez',", \\ 'Universidad Nacional de General Sarmiento (UNGS), Buenos Aires - Argentina \\ "Consejo Nacional de Investigaciones Científicas y Técnicas (CONICET), \\ Buenos Aires-Argentina
}

RESUMEN - La Historia Enseñada: una mirada a los cambios en las permanencias. Este trabajo explora la historia enseñada en la actualidad en el nivel secundario en Argentina, analizando especialmente las actividades y tareas que proponen y realizan cotidianamente en las aulas. La intención es subrayar los cambios y transformaciones de esta disciplina escolar tomando - aunque suene paradójico - sus haceres más recurrentes y persistentes. Se mostrarán las variaciones en las formas que adoptan, los contenidos que vehiculizan, la materialidad en que se basan, las representaciones que evidencian, y los sentidos a los que se asocian.

Palabras-clave: Enseñanza de Historia. Actividades. Disciplina Escolar.

ABSTRACT - History Teaching: a glance at the changes in the permanence. This paper explores the history taught nowadays in K-12 education in Argentina and analyzes the activities and tasks proposed every day in the classroom. The intention is to highlight the changes and transformations in this school subject, taking - paradoxically - its most recurrent and persistent tasks. The work shows the changes in the forms, contents, materials, representations and meanings associated to them.

Keywords: History Teaching. Activities. School Subject.

Educação \& Realidade, Porto Alegre, v. 42, n. 2, p. 747-769, abr./jun. 2017. 747 http://dx.doi.org/10.1590/2175-623658035 
Este trabajo analiza algunas características de la historia enseñada en la actualidad en el nivel secundario en Argentina, tomando especialmente las actividades y tareas que proponen y realizan cotidianamente en las aulas ${ }^{1}$. La intención es subrayar las transformaciones de esta disciplina escolar tomando, aunque parezca paradójico, sus haceres más recurrentes y persistentes.

Al respecto, en otro trabajo se ha señalado que las actividades más repetidas en las aulas de historia son las exposiciones, los cuestionarios y las síntesis (Gonzalez, 2013). Tales evidencias podrían ser tomadas como muestra de la persistencia de las rutinas inherentes al "código disciplinar" de la historia escolar (Cuesta, 1997). No obstante lo anterior, este trabajo se propone exponer que esas actividades recurrentes, si bien muestran tradiciones y permanencias, también manifiestan variaciones por las formas que adoptan, los contenidos que vehiculizan, la materialidad en que se basan, las representaciones que evidencian, y los sentidos a los que se asocian.

Se discutirá, así, la mirada que habla de la escuela como un espacio anacrónico y sin cambios al proponer una interpretación de la enseñanza de la historia como práctica cultural, y a las actividades áulicas como parte de una disciplina que es producto de la cultura escolar. Esto permitirá explicar las permanencias pero también advertir los cambios en lo que - a simple vista - no cambia, señalando transformaciones sutiles pero bastas.

Para cerrar, se planteará la necesidad de conocer (y reconocer) los haceres cotidianos en la enseñanza de la historia para fortalecer la formación docente.

\section{Una Mirada para Discutir y una Perspectiva para Ensayar}

Resulta un lugar común que la escuela es un espacio anacrónico y sin cambios ${ }^{2}$ Y, sin embargo, se suele criticar lo que no se conoce ya que, como señala Anne Marie Chartier (2000), los “haceres ordinarios”, las prácticas escolares y docentes cotidianas, son generalmente estudiadas de manera incidental o indirecta y hasta ignoradas por la investigación educativa.

En efecto, (al menos en nuestro país y nuestra disciplina) las prácticas de enseñanza constituyen un campo poco explorado y la mayoría de los estudios están basados en fuentes producidas fuera de la escue$\mathrm{la}^{3}$. Leyes, diseños curriculares y libros escolares han sido los objetos más analizados por la investigación educativa sobre la enseñanza de la historia con el objetivo principal de estudiar contenidos y sentidos de esa asignatura (Gonzalez, 2014b) ${ }^{4}$.

Esta falta de investigaciones sobre las prácticas desde las aulas podría explicarse por problemas teóricos y prácticos. En tal sentido, la escasez de recursos para sostener trabajos de campo de cariz etnográfico, las dificultades que conlleva acceder a observar clases y la complejidad de analizar la enseñanza atravesada por la multidimensionalidad, la simultaneidad, la inmediatez, la imprevisibilidad, la publicidad y la 
historia - tal como sintetiza Torres Santomé (1996), hacen que muchos de los esfuerzos en esta línea sean acotados.

Pero no es sólo un problema de recursos y fuentes: incluso algunos estudios que toman evidencias escolares juzgan saberes y prácticas desde las disposiciones oficiales y las sugerencias pedagógicas. Así, abundan evaluaciones (más que interpretaciones) que señalan "correspondencias y desviaciones con respecto a una prescripción u orientación" (Finocchio, 2003, p. 83). Pero la práctica docente cotidiana tiene temporalidades, lógicas e intereses diferentes a los que se derivan de los diseños curriculares, los materiales educativos o las propuestas didácticas. Por lo dicho, resulta necesario mirar la enseñanza de la historia desde las prácticas áulicas, en su día a día, tanto como adoptar una perspectiva que nos permita advertir su temporalidad y su naturaleza.

En relación con pensar las prácticas de enseñanza, resultan interesantes los aportes de Roger Chartier (1996). Este autor invita a pensar las prácticas no sólo como acciones sino como gestos, conductas, sentidos, ideas y emociones y a atender a su temporalidad, contexto e historicidad.

Con relación a la exploración de la enseñanza en las aulas, resulta relevante el estudio sobre las disciplinas escolares de Chervel (1991). Este investigador señala que, para comprenderlas, es necesario explorar tres cuestiones: origen, función y funcionamiento. Para el autor, el origen alude a los saberes que una disciplina transmite mientras que la función indica las finalidades que se le han asignado. En relación con el funcionamiento, Chervel sugiere que una mirada de largo plazo permite ver que las disciplinas escolares están constituidas "por una combinación, en proporciones variables según los casos, de diversos elementos: una enseñanza de exposición, ejercicios, prácticas de motivación e incitación al estudio y un aparato docimológico" (Chervel, 1991, p. 94). Y concretamente, en relación con las actividades, apunta: "si los contenidos explícitos constituyen el eje central de la disciplina enseñada, el ejercicio de ellos es la contrapartida casi indispensable [...] Sin ejercicio, y sin el correspondiente control de éste, no es posible fijar una disciplina" (Chervel, 1991, p. 90). Lo dicho por Chervel justifica el interés por las actividades, tareas, ejercicios, gestos y rutinas, es decir, por las prácticas que se realizan en las aulas al tiempo que anticipa la necesidad de observar lo cotidiano con un enfoque temporal amplio.

La necesidad de una perspectiva temporal extensa se combina con la exigencia de una exploración en detalle de las prácticas. Y, al respecto, resulta sugerente la aproximación a la "invención de lo cotidiano" de Michel de Certeau (2007) ${ }^{5}$. Este autor propone considerar lo cotidiano como ámbito de poiesis, y a las prácticas como "artes de hacer": "lo cotidiano se inventa con mil maneras de cazar furtivamente" (De Certeau, 2007, p. XLII). La idea de "cacería furtiva" atiende a lo que hacen los lectores con los textos que consumen, un consumo que es productivo, una producción que es silenciosa e invisible. Y esto permite comprender la relación y la diferencia entre, por un lado, las estrategias que desde un lugar de poder intentan fijar un sentido (en nuestro caso, leyes, norma- 
tivas, diseños curriculares, orientaciones didácticas), y, por otro, las tácticas, las apropiaciones plurales y móviles de los lectores (por ejemplo, los profesores) que les dan usos propios, con rechazos, distorsiones y artimañas, cuya significación no es necesariamente la resistencia sino el aprovechamiento, el gusto, la operatividad y la ocasión.

\begin{abstract}
A una producción racionalizada, expansionista, centralizada, espectacular y ruidosa, hace frente una producción de tipo totalmente diferente, calificada de 'consumo', que tiene como características sus ardides, su desmoronamiento al capricho de las ocasiones, sus cacerías furtivas, su clandestinidad, su murmullo incansable, en suma, una especie de invisibilidad pues no se distingue casi nada por productos propios (¿dónde tendría su lugar?), sino por el arte de utilizar los que le son impuestos (De Certeau, 2007, p. 37-38).
\end{abstract}

En diálogo con lo anterior, la categoría "cultura escolar" también permite pensar lo cotidiano, tanto en lo que se repite como en lo que se inventa cotidianamente. Según Viñao (2002, p. 59), la cultura escolar estaría constituida "[...] por un conjunto de teorías, ideas, principios, normas, pautas, rituales, inercias, hábitos y prácticas" sedimentadas a lo largo del tiempo en forma de tradiciones y regularidades compartidas en las instituciones educativas y que son transmitidas de generación en generación. Según este autor, sus rasgos característicos serían "la continuidad y persistencia en el tiempo, su institucionalización y una relativa autonomía que le permite generar productos específicos como las disciplinas escolares".

La definición de Viñao subraya las resistencias a los cambios por parte de la escuela, las tradiciones y regularidades sedimentadas en la "gramática escolar" estudiada por Tyack y Cuban (2001), y sus invariantes transmitidas en la "forma escolar" (Vincent; Lahire; Thin, 1994). Sin embargo, como señala al final de su propia definición, la cultura escolar posibilita considerar el carácter inventivo y productivo de la escuela, no sólo como lugar de recepción de prescripciones y orientaciones para la enseñanza, sino como espacio para la generación de productos específicos: las disciplinas escolares. Así, entonces, esta categoría también permite percibir la naturaleza propia y específica de las prácticas escolares y entender a la escuela como un lugar de producción de una cultura (Chervel, 1992).

La perspectiva teórica hasta aquí sintetizada es la que se ha utilizado para este acercamiento a la historia enseñada que se expondrá - en parte - a continuación. Desde el punto de vista empírico, se han utilizado un conjunto de carpetas ${ }^{6}$ de alumnos de secundaria y una serie de observaciones de clases realizadas en escuelas medias públicas y privadas de la Provincia de Buenos Aires ${ }^{7}$. Esto responde a la necesidad de realizar un acercamiento múltiple para reconstruir y analizar la historia enseñada en la actualidad, un acercamiento que, desde luego, no se puede realizar solo a partir de "pruebas visuales" (como las observaciones directas) sino que es posible en el cruce de diversas fuentes ${ }^{8}$.

$750 \quad$ Educação \& Realidade, Porto Alegre, v. 42, n. 2, p. 747-769, abr./jun. 2017. 
Antes de la presentación de las evidencias, resulta necesario realizar dos advertencias. Por un lado, este trabajo no supone conclusiones taxativas y generalizables. Se aludirá a una serie de transformaciones en la historia enseñada como tendencias que se combinan - con mayor o menor grado de intensidad o fragilidad - dando lugar a prácticas diversas. Por otro, resulta inevitable hacer mención a la desigualdad que caracteriza la realidad educativa argentina actual en general y a la región donde se desarrolla esta indagación en particular. Indudablemente, esta cuestión atraviesa a las escuelas y las aulas, y se ha podido observar en el trabajo de campo. No obstante, también se ha podido advertir que - más allá de lo público o lo privado, de las diferencias socioeconómicas, de la fragmentación y desigualdad escolar - las diferencias no se juegan tanto en la materialidad como en las apropiaciones, en las posibilidades como en las expectativas, en las condiciones como en las representaciones (por ejemplo en las miradas y expectativas que los docentes tienen de sus estudiantes, de su oficio, de su propia práctica, etc.). Desde luego, se trata de una cuestión sensible y compleja que ameritaría un desarrollo más extenso y una reflexión más pausada. Sin embargo, y como se verá más adelante, las actividades recurrentes aparecen en distintos tipos de escuelas sean públicas o privadas, atiendan a sectores socioeconómicos favorecidos o desfavorecidos ${ }^{9}$.

\section{Algunas Evidencias para Analizar}

Como se ha dicho, en este trabajo sobre la historia enseñada se recortarán y analizarán los haceres recurrentes, es decir, aquellos que aparecen en todas las aulas de historia (dando cuenta de un núcleo duro y perenne del código disciplinar) pero para señalar las mudanzas que también permiten vislumbrar.

Una mirada como la que se propone, es decir observar los cambios en las permanencias, supone una comparación con la historia enseñada entre fines del siglo XIX y casi hasta fines del siglo XX que, a grandes rasgos, ha sido definida como libresca, elitista, memorística, expositiva y concentrada en saberes de tipo fáctico - político, una historia con objetivos civilizatorios y patrióticos. Desde luego, se trata de una caracterización que subraya las tendencias más fuertes por aquel entonces, sin desconocer la multiplicidad de prácticas (pretendidas y llevadas a cabo) en la historia de la enseñanza de la historia ${ }^{10}$.

Tales características fueron objeto de reforma cuando se sancionó en Argentina la Ley Federal de Educación 24195 en 1993 que propuso una historia escolar para formar ciudadanos democráticos y buscó actualizar los contenidos a través de la ponderación de la historia social más conceptual y problematizadora, la inclusión de procedimientos y principios explicativos propios de las ciencias sociales, y la jerarquización de la historia contemporánea y reciente (Finocchio, 1999; De Amézola, 2008). A esto se suma que, por las transformaciones comunicativas de los últimos años, se registra el uso de diversos materiales que amplían el repertorio de lo escrito e impreso a lo audiovisual y multimedial. 
A continuación, presentaré el análisis incluyendo algunos pocos extractos de las fuentes con un afán más ilustrativo que demostrativo ${ }^{11}$.

\section{Exposiciones}

En todas las aulas observadas, se ha podido ver profesores realizando exposiciones y explicaciones orales, una práctica que no aparece prescrita en la normativa educativa en vigencia ni es especialmente fomentada por las orientaciones didácticas actuales ${ }^{12}$. Sin embargo, y como señala el ya citado Chervel (1991), la exposición de los docentes es uno de los elementos constitutivos de las disciplinas escolares por lo que su persistencia daría cuenta de la "cultura escolar".

No obstante, en esta actividad recurrente - que $a$ priori estaría señalando la persistencia de prácticas conocidas - se encuentran indicios de cambios en las formas, los vínculos pedagógicos, en los modos de entender el aprendizaje, las subjetividades, los contenidos desplegados, las materialidades que acompañan la oralidad, en el modo de concebir la historia y su enseñanza, entre otros.

El modo exponer, más propenso a la exposición dialogada que al monólogo, la forma de apelar a los estudiantes, de invitarlos a participar, de estar atentos al feedback, de pedirles que justifiquen sus afirmaciones, que discutan perspectivas, que se animen a realizar inferencias, que construyan conocimiento de manera colectiva son algunos rasgos salientes. Esto habla, desde las aulas de historia, de la transformación general de los vínculos pedagógicos y el papel activo de los estudiantes aun en un hacer que parece ser potestad exclusiva del profesor.

Muchas exposiciones de los profesores manifiestan una insistente preocupación por conocer y trabajar con los saberes previos de los estudiantes, en anclar el nuevo contenido en la trama cognitiva de los jóvenes. En este sentido, la perspectiva constructivista y ausubeliana, instalada desde el discurso pedagógico a partir de la reforma de los '90, transmitida en la formación docente inicial y continua así como en la bibliografía didáctica de aquellos años, parece haber llegado a las au$\operatorname{las}^{13}$. En las siguientes transcripciones ${ }^{14}$ (ejemplos 1 y 2), los docentes inician la secuencia didáctica apelando a la definición de los conceptos recurriendo a las representaciones y saberes previos de los estudiantes.

$\mathrm{P}:[. .$.$] entonces lo que vamos a ver en el cercano oriente es una zona lla-$ mada Mesopotamia xxx ¿Qué significa la palabra Mesopotamia? [...] A ver, en Argentina hay una región, a ver, hay una región que se conoce como Mesopotamia, de hecho uno cuando estudia cuando es más chico las distintas regiones, una región que es la Mesopotamia que incluye iqué provincias?

A: Las del sur.

A: Entre Ríos.

A: Córdoba.

P. Noooo $[\ldots]$

A: Corrientes.

P: Corrientes, Entre Ríos, Misiones. A ver, ubiquemos en el mapa. Escuchen. Ubiquemos en el mapa mental nuestro Entre Ríos, Corrientes y

752 Educação \& Realidade, Porto Alegre, v. 42, n. 2, p. 747-769, abr./jun. 2017. 
Misiones. ¿Están entre qué?

A: ¡Ríos!

P: ¡Entre ríos! ¿ ¿í? La palabra Mesopotamia significa xxxxx entre ríos. [...] en Argentina tenemos una región.... que se denomina Mesopotamia que son tres provincias que están rodeadas por ríos. En el caso del Cercano Oriente vamos a encontrar una región que también se llama Mesopotamia $[\ldots]$

(Ejemplo 1, Transcripción de observación de clase en ler año, escuela privada, 2012).

P.: Hoy vamos a comenzar a ver el proceso de descolonización, que sucede después de la segunda guerra mundial ¿alguien se acuerda qué era el imperialismo?_Si yo les digo "descolonización”, ¿̇cómo lo definirían en una palabra?

(Ejemplo 2, Transcripción de observación de clase en 5to año, escuela pública, 2014).

Por otra parte, la preocupación por hacer que las explicaciones sean significativas para los estudiantes, hace que varios profesores recurran a analogías, comparaciones y referencias al presente (y futuro) de los jóvenes, haciendo que "lo extraño se vuelva familiar" (Monteiro, 2005) y generando oportunidades para la co-construcción de sentidos y significados.

P. Exacto. ¿Y cómo se recuerda, dice Traverso? Es subjetiva y es singular (la profesora va anotando en el pizarrón)[...]En relación al tiempo, Traverso dice que siempre se recuerda en presente. ¿Por qué dice que siempre se recuerda en presente? [...]“Siempre recordamos desde el presente”, dice.

(Los alumnos releen el texto)

A5: depende de la situación en la se encuentre, va a recordar el mismo hecho, de una u otra forma

P: Exactamente. Que es lo que está diciendo xxx ¿sí? El tiempo va modificando el recuerdo. Por eso siempre recordamos en presente ¿isí? No recordamos las cosas tal cual ocurrieron sino tal cual justamente las vamos recordando.

A6: Todos recuerdan cosas distintas

P: También! Por eso es singular. Es lo que hablamos siempre. El año que viene, cuando se reúnan y hagan la fiesta del reencuentro de los egresados 2013, no todos van a recordar lo mismo de este año. isí? Ni todos van a recordar $=$ con las mismas características a las mismas personas=

A: $=\operatorname{xxxx}$ (los alumnos hacen comentarios y ríen)=

P: Por eso es singular y subjetiva[...] ¿Y la historia?[... ]Porque lo que quiere hacer Traverso es marcar la diferencia que hay entre historia y memoria... A7: La historia es =objetiva $=$

A?: =subjetiva $=$

A8: $=$ sub $=$ no!

A4: =intenta $=$ ser objetiva

A6: $=$ se supone $=$ que tiene que ser objetiva

A?: =trata $=$

P: Ojo con esto! Acuérdense [...] que la objetividad absoluta [...] =no existe $=$.

A4: $=$ no existe $=$

(Ejemplo 3, Transcripción de observación de clase en 6to año, escuela privada, 2013). 
Asimismo, la subjetividad (valores, ideologías, posicionamientos) de los profesores se cuela en lo que exponen y cómo lo hacen: mencionan sus perspectivas, sus lecturas, sus modos de acercarse a lo que están transmitiendo al tiempo que generan puentes con el presente de los estudiantes. Véase en este ejemplo (n4) cómo el docente alude a su propia biografía y apela a las de sus estudiantes.

$\mathrm{P}:[. .$.$] hablen con personas mayores de setenta años y van a ver qué re-$ cuerdan de Perón. Lo mismo pasa ahora. ¿Quién les dio la primera netbook?

A: Cristina.

P: Mi padre siempre recuerda que le llegó su primera pelota y su guardapolvo al Chaco por Perón.

(Ejemplo 4, Transcripción de observación de clase en 5to año, escuela pública, 2012).

Las exposiciones también dejan a la vista las mudanzas en los contenidos enseñados (que se relacionan con las transformaciones de los contenidos prescritos en los diseños curriculares). Si bien la dimensión política y temporal sigue siendo el eje ordenador de lo trabajado en el aula (tal como también sucede con los diseños), las perspectivas sobre la historia y su enseñanza han mutado. En lugar de ponderar los acontecimientos (aunque no se los desconoce) se jerarquizan los procesos y conceptos a la vez que se trabaja con múltiples causas y consecuencias (como en el ejemplo 5 donde el docente realiza una explicación de las causas de la Revolución de Mayo apelando a la participación de sus alumnos)

P: Bueno parece que la noticia de la caída de la Junta de Sevilla fue el detonante. ¿Es la única causa?

A: Esa es la causa más inmediata

P: Pero hay causas que vienen de mucho más atrás. ¿Alguien se acuerda alguna?

A: El iluminismo

P: El iluminismo

A: La Revolución de Estados Unidos

P: Podría ser una causa.

A: Napoleón

P: Podría ser Napoleón. ¿Alguna más? [...]

(Ejemplo 5, Transcripción de observación de clase en 3er. año, escuela privada, 2013).

En el mismo sentido, muchas exposiciones de los docentes incluyen interrogantes que los profesores lanzan a sus estudiantes para problematizar y discutir (como en el próximo ejemplo donde la docente cierra el trabajo con los conflictos y situaciones de la "guerra fría"). Esto también daría cuenta de cambios en los sentidos de la historia a enseñar, más propensa a las preguntas y problemas que a la repetición de datos y fechas.

P: entonces, a partir de lo que estuvimos analizando de América latina y África, ¿̇podemos matizar la idea de un mundo bipolar?

(Ejemplo 6, 5to año, escuela pública, 2014). 
Asimismo, en las exposiciones dialogadas también aparecen indicios que muestran que en las aulas se trabaja con la idea de la historia como lectura del tiempo pasado. Los profesores introducen menciones sobre el trabajo historiográfico, a la multiperspectividad en las interpretaciones, al trabajo de algunos historiadores e incluso de su valoración sobre sus aportes. Esto fue especialmente subrayado en tiempos de la reforma educativa, cuando se instalaron como novedad los contenidos procedimentales (Finocchio, 1999) acercando la historia escolar a la historia académica.

Para cerrar este análisis de las exposiciones de los profesores parece interesante señalar que, en tiempos en los que el saber está descentrado, circulando por muy diversos canales, cuando los saberes ya no son "propiedad" exclusiva de los profesores, las aulas siguen convocando y colocando a los docentes frente a la responsabilidad de enseñar, explicar, orientar, acompañar, transmitir.

\section{Cuestionarios}

Los cuestionarios de preguntas también aparecen como un elemento recurrente en las clases observadas y en las carpetas de estudiantes analizadas. Desde luego, las preguntas y consignas para que los estudiantes contesten y resuelvan también aparecen en otras fuentes como en los libros de textos escolares y materiales didácticos diversos que son tomadas, reproducidas, recortadas, adaptadas o modificadas por los docentes.

Varios autores (por ejemplo, Aisemberg, 2005) señalan que esta actividad generalmente se estructura a través de "cuestionarios-guía" o "micro-cuestionarios" que incluyen preguntas analíticas y atomizadas que provocan que los alumnos sólo desplieguen estrategias de localización de la información necesaria para responder (lo que no garantizaría necesariamente la comprensión). Si bien esto aparece con claridad en las carpetas, no es lo único. En tal sentido, es destacable que esas fuentes también muestran que los cuestionarios solicitan que los estudiantes expliquen, relacionen, comparen y elaboren conclusiones.

El siguiente ejemplo $\left(n^{\circ} 7\right)$ muestra un conjunto de consignas a partir de las cuales los estudiantes deben explicar, realizar un informe, narrar, comparar y justificar.

1. Explica el modelo económico con el que se inserta a la Argentina en el mercado internacional

2. ¿Cómo se produce la expansión rural y la ganadería?

3. Realiza un informe sobre la inmigración extranjera, su localización y sus ocupaciones

4. Cuenta cómo se fueron conformando los barrios porteños

5. Compara la vida en los conventillos con la de la elite tradicional

6 . ¿Por qué este régimen político gobernante recibe el nombre de oligárquico?

(Ejemplo 7, transcripción de una carpeta de estudiante de escuela pública, $5^{\circ}$ año, 2009) 
Asimismo, los cuestionarios muestran que se estimula insistentemente a los estudiantes a dar sus opiniones y pareceres, a manifestar qué sienten, qué creen, cómo juzgan, qué piensan ${ }^{15}$

Lean el siguiente artículo periodístico y luego realicen las consignas: a-¿̨cuál creen que es la razón por la que se escribió este artículo? b-ipor qué creen que las bombas atómicas sobre el Japón son acontecimientos que tenemos que recordar?

c-Averigüen cuáles fueron los efectos inmediatos y tardíos de las bombas atómicas en la saludo de los sobrevivientes y en el ambiente

d-¿iPor qué creen que las bombas atómicas arrojadas sobre el Japón son comparadas con el genocidio alemán?

(Ejemplo 8, transcripción de una carpeta de estudiante de escuela pública, 5to año, 2011).

Primer gobierno de Hipólito Yrigoyen (1916-1922)

1. ¿Por qué crees que Yrigoyen pudo llegar a la presidencia? ¿cuál era la situación en la que asumía

2. ¿Qué estrategias implementó para poder gobernar?

3. ¿Cuál era su relación con los sectores medios? ¿cómo fue calificado por ello?

4. ¿Qué reclamaban los universitarios?

5. Realiza un informe describiendo la relación con los obreros describiendo particularmente dos hechos fundamentales: "La Patagonia rebelde" y la "Semana trágica"

(Ejemplo 9, transcripción de una carpeta de estudiante de escuela pública, 5to año, 2009).

A través de los ejemplos anteriores de cuestionarios es posible ver las continuidades y cambios. En otras palabras, si la forma aparece como algo rutinario y conocido, las demandas de sus consignas muestran variaciones.

Como en el caso de las exposiciones, los cuestionarios también muestran cambios en los contenidos de la historia enseñada, que actualmente toma en consideración, por ejemplo, diversos sujetos colectivos (véase las preguntas en relación con los sectores medios, estudiantes, obreros en los ejemplos 7 y 9). Asimismo, también dan indicios de los cambios en la materialidad de la enseñanza, mostrando que en las aulas circulan diversos recursos más allá de los textos escolares, como artículos periodísticos (ejemplo 8), recursos visuales, audiovisuales, etc.

\section{Sintesis}

Finalmente, las síntesis realizadas por profesores y estudiantes en diversos formatos también son actividades recurrentes.

En las clases y las carpetas son visibles los resúmenes escritos y las síntesis gráficas -cuadros sinópticos, esquemas y redes. En las clases observadas, esas sinopsis acompañan la exposición del profesor desde el pizarrón (o es armada a posteriori) y luego es copiada por los alumnos en las carpetas. En éstas, también se escriben resúmenes escritos que toman como fuente el libro de texto escolar, con el añadido de la "preo- 
cupación" insistente de que los alumnos escriban "con sus propias palabras" y "evitando copiar literalmente" (expresiones que se convierten en parte de muchas consignas).

En muchos casos, los esquemas toman formas gráficas construyendo secuencias derivativas (una idea general que se abre en múltiples ideas secundarias conexas). Nuevamente (como se citó para el caso de las exposiciones) esto parece dar cuenta de ciertas ideas que han tenido alto impacto en el imaginario pedagógico post-reforma educativa en Argentina: que el aprendizaje significativo - conforme a la teoría de Ausubel - se produce por asimilación de contenidos en sucesivo grado de diferenciación y especificación ${ }^{16}$. En otros, los esquemas sintetizan con palabras claves algunas ideas que exponen en un cuadro sin estar acompañadas por flechas que manifiesten vinculaciones.

De cualquier modo, una mirada atenta a esos cuadros y gráficos muestra que los mismos incluyen conceptos además de datos, procesos más que acontecimientos, y definiciones historiográficas por lo que como en el caso de las exposiciones - podemos tomarlos como rastro de una historia más explicativa.

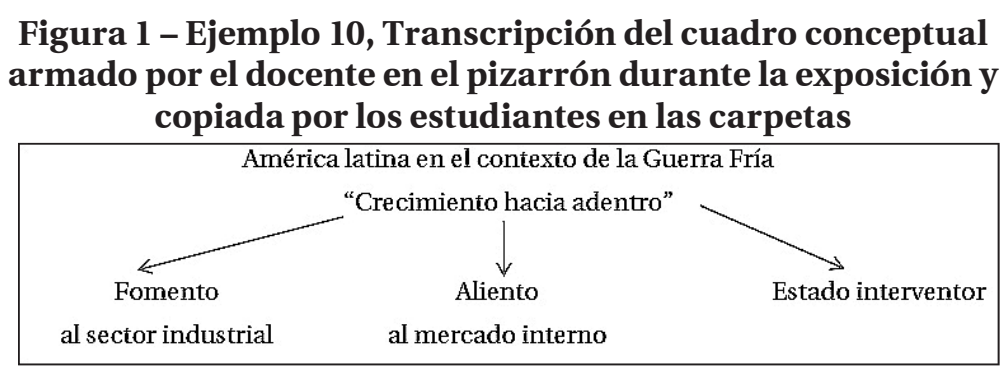

Fuente: Ejemplo Escuela pública, 5to año, 2014.

Otras síntesis adoptan la forma de "cuadros de llave", "cuadros comparativos de doble entrada”, etc. e incluso al inicio del año se copian en las carpetas las "técnicas de estudio"17.

En las carpetas, además, se ven resúmenes escritos que realizan los estudiantes como tareas (y cuya fuente es un texto o libro escolar) o recapitulaciones que los profesores dictan luego de la explicación oral ${ }^{18}$.

Pero, además, las síntesis no sólo se realizan en el pizarrón y se copian en las carpetas. En ocasiones, quedan plasmadas en láminas que quedan colgadas en las paredes de las aulas o en pasillos de la escuela, a fin de exponer el trabajo en clase ${ }^{19}$. Nuevamente (como en el caso de las exposiciones de los docentes), la confección de láminas no aparece señalada en las fuentes normativas ni es ponderada en la bibliografía didáctica. Así, las láminas también demuestran la potencia de la cultura escolar, es decir, mostrando tradiciones, regularidades y reglas de juego que se transmiten de generación en generación como señalara Viñao (2002). Pero, aunque parezcan una evidencia de las herencias y repeticiones, también se ven los cambios: las láminas tienen un papel importante como expresión del trabajo de los alumnos pero ya no son 
un elemento para la apreciación sensible, para brindar la base del conocimiento (Feldman, 2004) sino que son resignificadas en un contexto en que la imagen tiene gran centralidad en el mundo contemporáneo (Finocchio, 2011).

Para cerrar este apartado, se apuntarán algunas ideas finales.

Por un lado, en torno a los haceres recurrentes, y en esta comparación que se planteaba con las prácticas del siglo XX, resulta interesante no sólo señalar lo que se ve sino también apuntar lo que no se ve en las aulas. Y lo que no se ve es la repetición de datos fácticos de memoria y utilización exclusiva del libro de texto escolar para repetir la lección oral. Tales prácticas fueron documentadas en informes de observaciones de aulas de historia en el nivel medio a principios del siglo XX.

[...] en Historia, se aprenden prolijas enumeraciones de dinastías, nombres de batallas y de jefes que tomaron parte en ellas, fecha, número de combatientes y uno que otro episodio considerado de importancia [...] y todo ello dicho casi siempre hasta con las mismas palabras del texto, sin vida, de una manera mecánica [...] aumentando así el número de palabras, no digo ideas, que almacena transitoriamente el alumno-maestro o el bachiller, para olvidarlas, naturalmente, después del examen. Informe de Pablo Pizzurno (MJIP, 1902, p. 6).

[...] el método tradicionalmente usado en nuestros institutos secundarios, públicos o privados, para la enseñanza de la Historia, consiste en adoptar un manual nacional o extranjero como libro de texto, y en obligar a los alumnos a estudiarlo, por dosis en sus casas, para recitarlo en clase ante los condiscípulos y el Profesor, quien suele agregar algunos comentarios o ampliaciones. Nada más contrario a los buenos principios pedagógicos que el método mecánico indicado [...] La reacción debe ser [...] sustituirlo por el llamado (método) activo, experimental o intuitivo. [...] El rasgo central del método que preconizo consiste en objetivar la enseñanza, en volverla práctica, realista; en hacer del alumno, no un ente pasivo, como en la actualidad, sino en un sujeto activo que observa, que compara, que raciocina, induciendo o deduciendo, que juzga, que colabora de una manera principal en la obra de adquisición de conocimiento (Informe de Antonio Dellepiane) (MJIP, 1903, p. 61-65).

Desde la investigación en curso es posible señalar que las dos prácticas señaladas (y criticadas) por Pizzurno y Dellepiane no se ven en las aulas de principios del siglo XXI. Y estas ausencias también dicen mucho de los cambios. Si bien la exposición oral persiste, ya no es más esa lección oral al frente parado junto al pizarrón. Se trata, en contrapartida, de una puesta en común y grupal del trabajo realizado también grupalmente. Y si la lección persiste, la forma cambia: los alumnos, sentados al lado del escritorio del profesor, dan "oral para levantar nota" mientras el resto del grupo hace otra tarea. Del mismo modo, y para el caso del texto escolar, aun cuando éste sigue vigente en las aulas, 
se presenta en diversos formatos (dossier, fotocopias, hojas sueltas) y combinado con otros materiales escritos (artículos periodísticos, textos académicos, etc.), visuales y audiovisuales.

Por otro lado, y volviendo a las advertencias comentadas al inicio del trabajo, resulta claro que los cambios en lo que persiste - como se ha mostrado - no son generalizables y que la desigualdad educativa atraviesa la historia enseñada. No obstante, la indagación hasta aquí desarrollada permite señalar que estas prácticas se repiten en aulas de escuelas diversas ${ }^{20}$. Dicho en otras palabras, los profesores exponen y explican en distintos tipos de escuelas. Del mismo modo, tanto la carpeta de un estudiante de una escuela privada orientada a sectores altos y como la que pertenece a un alumno de una escuela pública de un barrio de sectores bajos contienen cuestionarios y síntesis.

Sobre los cambios en las permanencias, y como se ha dicho, no es posible generalizar. Desde luego, hay profesores que exponen monologando, cuestionarios que buscan solo que los estudiantes reproduzcan información y no que expliquen problemas, así como síntesis que repiten de manera desordenada un conjunto de datos sin vinculaciones. Empero, en la mayoría de las clases observadas y carpetas analizadas he podido advertir las mutaciones señaladas lo que me permite confiar en que se trata de mudanzas férreas y no prácticas aisladas de algunos profesores.

Y es que toda práctica (como la práctica docente) nunca es irreductiblemente individual, sino que es tan personal como colectiva, $\tan$ singular como plural. Como señala Chartier (2007) no existen recepciones o producciones puramente subjetivas e individuales sino supuestos pertinentes, prácticas significativas y propósitos relevantes, que permiten explicar los haceres convergentes y análogos. Este mismo autor señala que la manera en que los actores sociales otorgan sentido a sus prácticas y a sus enunciados se ubica en la tensión entre, por un lado, las capacidades inventivas de los individuos o las comunidades y, por otro, las restricciones y las convenciones que limitan lo que les es posible pensar, decir y hacer. Esto es válido, según Chartier, tanto para las obras letradas y creaciones estéticas como para las prácticas ordinarias, diseminadas y silenciosas que inventan lo cotidiano. En este caso, resulta sugerente para interpretar las prácticas en la historia enseñada.

\section{A Modo de Balance y otros Apuntes para Continuar}

Como se ha intentado poner de manifiesto hasta aquí, la historia enseñada muestra haceres que forman parte del repertorio histórico de las disciplinas escolares, dando cuenta de la potencia de la cultura escolar, de la persistencia de prácticas que circulan en la escuela, que son transmitidas de generación en generación, que no se ponen en entredicho y que se muestran desacompasadas de lo prescrito en la normativa o de lo sugerido en la didáctica.

A la vez, esos mismos haceres recurrentes y persistentes son los que permiten afirmar que hay cambios importantes. En efecto, expo- 
siciones, cuestionarios y síntesis dan cuenta de mutaciones en relación con los vínculos, las formas, los saberes, la materialidad y los sentidos de la historia en las aulas a principios del siglo XXI. De allí la citada necesidad de una mirada en perspectiva temporal amplia, puesto que estos aspectos fueron ponderados en la reforma de los ' 90 antes descrita.

La participación activa de los estudiantes aún en una práctica que solo parece mostrar el protagonismo de los profesores - como es la exposición - como así también en el énfasis en que los estudiantes opinen y den sus pareceres frente a ciertos procesos históricos muestran cambios en los vínculos pedagógicos, en la forma de entender la enseñanza y el aprendizaje. Las síntesis en forma de esquemas y gráficos también mostrarían algo de esa sociabilidad cada vez más estructurada por la imagen (Mafessoli, 1998 apud Finocchio, 2011, p. 189). Por otra parte, todos estos haceres evidencian - cada uno a su modo - los cambios en los contenidos y en la materialidad de la enseñanza de la historia hoy: procesos y conceptos por sobre hechos y datos así como el uso y apropiación de diversos materiales más allá del libro de texto, muestran mutaciones silenciosas y sutiles pero no menos importantes.

Ahora bien, frente a las evidencias hasta aquí presentadas, es pertinente plantear dos preguntas ìpor qué persisten y por qué se repiten tanto? Como se anticipó, inmovilismo y conservadurismo de la cultura escolar y de las prácticas de enseñanza no resultan respuestas satisfactorias.

La perspectiva de la invención de lo cotidiano puede servir para entender mejor las persistencias. Y es que "[...] la vida cotidiana necesita de un orden contenedor, que permita operar en el día a día con cierta previsibilidad. Entonces, la reiteración de sucesos y prácticas en el tiempo es su sustento: rutinización, historicidad y memoria" (Maronna; Sanchez Vilela, 2006, p. 97). A simple vista, la vida cotidiana transcurre con una apariencia de inmovilidad y quietismo. Y, en parte, es real que los sujetos construyen, interiorizan y repiten día a día hábitos, gestos y prácticas. Pero en la aparente estabilidad, se producen cambios permanentemente " $[\ldots]$ el orden cotidiano se mueve pendularmente entre la conservación y el cambio. Es un movimiento casi imperceptible, no es estruendoso, pero actúa transformando, de modo constante, la realidad" (Maronna; Sanchez Vilela, 2006, p. 104). Y lo cierto es que, en lo cotidiano, los cambios son demasiado pequeños para notarlos, no se perciben en lo que vemos todos los días, pero sí se llegan a ver si se nos presenta una fotografía antigua con la que contrastar. De allí que se haya optado por comparar los haceres actuales con los que se presentaban mayormente entre el siglo XIX y mediados del siglo XX.

El sentido de las persistencias y continuidades también es analizado por Chervel (1991, p. 84) quien entiende que la estabilidad de una disciplina escolar "[...] no es, pues, como se piensa a menudo, un efecto de la rutina, del inmovilismo, de la pesadez o de la inercia inherentes a la institución, sino que es el resultado de un largo proceso de concertación basado en el aprovechamiento común de una considerable experiencia pedagógica".

760 Educação \& Realidade, Porto Alegre, v. 42, n. 2, p. 747-769, abr./jun. 2017. 
Esta última idea de "experiencia pedagógica” acumulada permite plantear la pertinencia de indagar los haceres cotidianos para fortalecer la formación docente, cuestión sugerida por Anne Marie Chartier (2000). Esta autora señala que las prácticas ordinarias de los docentes no sólo son variables no documentadas por la mayoría de las investigaciones educativas sino que además, generalmente, son pensadas como las banalidades de las reiteraciones prácticas, consideradas insignificantes y triviales. Por lo mismo, según la autora, esos haceres son ignorados por las instituciones de formación docente que están menos preocupadas en transmitir las tácticas elementales y más abocadas a anunciar la renovación de las conductas pedagógicas o didácticas. Y, para esta investigadora, indagar esos haceres ordinarios resulta fundamental para la formación docente por varios motivos: para legitimar a profesores y maestros como expertos, y para habilitar márgenes de libertad frente a esquemas de intervención obligatorios.

Conocer y reconocer los haceres cotidianos en la enseñanza de la historia, incluso con sus rutinas, puede fortalecer la formación de profesores y consecuentemente (aunque suene paradójico) renovar la enseñanza puesto que permite acercarse a la naturaleza de las prácticas, sus transformaciones silenciosas y sus posibilidades de cambio. Y esto es particularmente relevante para la formación docente en historia puesto que supone pensar las prácticas históricamente y discutir el repetido discurso acerca del "fracaso de las reformas". Por un lado, porque al colocar las transformaciones en una temporalidad más amplia, se puede advertir cómo las mutaciones fueron decantando y han sido incorporadas a las aulas. Por otro porque, al pensar las prácticas como apropiaciones, como tácticas de significación activas, se comprende mejor que las normas nunca son incorporadas de acuerdo con su formulación original sino que son recibidas y reinterpretadas de acuerdo a las tradiciones pedagógicas. De allí que, a pesar que actualmente la norma oficial no las incluya o las pondere, las exposiciones, cuestionarios y síntesis continúen realizándose aunque incorporando otras mudanzas sí señaladas en esas prescripciones.

Como se ha dicho, el desconocimiento de los haceres cotidianos, la negación de la historicidad de las prácticas y el olvido de sus temporalidades mixturadas y diversas (desacompasadas de lo normativo y pedagógico) están en la base de esa mirada que ve una escuela que no cambia y profesores que no innovan. Una perspectiva así es por demás problemática y estéril puesto que los docentes se sienten juzgados, solos e inermes frente a una tarea que no sólo no es reconocida en su complejidad sino que además es catalogada de inconducente y banal, desviada de la norma y atrasada en relación con las teorías didácticas.

Ahora bien, y aun asumiendo que la escuela y la enseñanza de la historia deben cambiar, cabe preguntarse ¿las prácticas cambian de un día para otro? Si en verdad se cree que la enseñanza debe renovarse, ¿no debería tomarse como punto de partida lo que se hace cotidianamente sin degradarlo o descartarlo? ¿No será que en la escuela y la enseñanza se necesitan rutinas? ¿No será que se debe explorar, analizar y compren- 
der las rutinas como parte constitutiva de un “código disciplinar” necesario para posibilitar la transmisión cultural a las jóvenes generaciones? Sobre esto último, Chervel (1991, p. 111) afirma:

\begin{abstract}
[...] las disciplinas escolares deben el papel, infravalorado pero considerable, que han desempeñado en la historia de la enseñanza y la historia de la cultura a las circunstancias en que se gestaron y a su organización interna. Fruto de un diálogo secular entre maestros y alumnos, las disciplinas constituyen hasta cierto punto el código que dos generaciones han elaborado lenta y minuciosamente de común acuerdo para permitir que una de ellas transmita a la otra una cultura determinada. La importancia de esta creación cultural está a la altura de lo que se pone en juego, pues se trata nada menos que de la perpetuación de la sociedad. Las disciplinas, por tanto, son el precio que la sociedad debe pagar a su cultura para poder transmitirla en el marco del colegio o de la escuela ${ }^{21}$.
\end{abstract}

Llegados a este punto, hay un detalle que quizás resulte obvio pero tal vez merezca algunas palabras. Con este trabajo no se está proponiendo una arenga contra el cambio; no se defienden las prácticas rutinarias solo porque sean parte de un cotidiano escolar que se desconoce. No quisiéramos que este escrito sirva para alentar la resistencia y el rechazo que muchos profesores manifiestan frente a lo que el campo pedagógico y didáctico puede aportar para pensar y repensar su tarea cotidiana (puesto que la práctica no es solo hacer sino también pensar sobre el hacer). Desde luego, tampoco desearíamos que las evidencias aquí presentadas sirvan para alimentar la perspectiva que atiende solo a lo que perdura en la escuela. Por el contrario, hay muchas revisiones por hacer en la enseñanza de la historia y las mismas podrían comenzar por estos haceres cotidianos desde la propia formación docente.

Lo que sí se plantea, siguiendo a De Certeau (2007), es que dar visibilidad a lo cotidiano permite hacer inteligibles y comprensibles las prácticas culturales, su carácter productivo e inquieto, su naturaleza y temporalidad, y, en consecuencia, la potencia y la posibilidad de sus cambios. Esto resulta especialmente sugerente para pensar la enseñanza de la historia porque, como señalara Jackson (1996, p. 197), “[...] el transcurso del progreso educativo se parece más al vuelo de una mariposa que a la trayectoria de una bala".

Por eso, se insiste en que los interrogantes y exploraciones serían más fructíferos desde la consideración de la enseñanza de la historia en las aulas como práctica cultural, lo cual nos acerca a su temporalidad mixturada, a su historicidad implícita, a su memoria tácita, a sus haceres invisibles...

Este trabajo fue un intento de avanzar en esta línea, mostrando mutaciones en lo que permanece, dando cuenta de los cambios en las rutinas, en fin, exponiendo algunas características y transformaciones en la historia enseñada a principios del siglo XXI.

Recibido en 24 de agosto de 2015 Aprobado en 03 de agosto de 2016 


\section{Notas}

1 Se planteará un acercamiento a la historia enseñada entendiendo que tal categoría permite captar los saberes y prácticas en las aulas. Al respecto, en la bibliografía sobre este tema, se registran diversas denominaciones. Por ejemplo, y para el caso de la historia de la enseñanza de la historia en España, Raimundo Cuesta (1997) alude a la "Historia regulada" (relacionada con la normativa), la "Historia soñada" (las propuestas de los grupos de renovación pedagógica) y la "Historia enseñada" (para remitir a "la que el profesorado practicó realmente en las aulas"). Este mismo autor señala también la importancia de atender a los "textos visibles", es decir, los discursos contenidos en los documentos, y los "textos invisibles", es decir, "los contextos escolares de la práctica de la enseñanza” (Cuesta, 1997, p. 21)

2 Esta idea es alimentada por especialistas del campo pedagógico y asumida por los propios profesores que dan cuenta que la nostalgia y la percepción de inmovilismo impregnan sus representaciones. Así, se explican las palabras de dos profesores durante el trabajo de campo, quienes que comentaron-palabras más, palabras menos - "que la enseñanza de la historia ya no es lo que era" o que "en las clases de historia se hacen las mismas cosas de siempre".

3 En Brasil, en cambio, hay una fructífera línea de pesquisa en torno a los saberes y prácticas en la enseñanza de la historia. Por ejemplo, Monteiro (2007); Monteiro, Gasparello y Magalhaes, (2004); Monteiro y Penna (2011) entre muchísimos otros.

4 Cabe mencionar como excepción el trabajo de Silvia Finocchio e Hilda Lanza (1993) llevado a cabo en Ciudad de Buenos Aires -antes de la reforma abierta por la sanción de la Ley Federal de Educación- en la que se realizaron observaciones de clases y entrevistas a profesores a fin de caracterizar los ámbitos que constituyen el discurso de los profesores de historia del nivel medio. Entre otros trabajos posteriores sobre saberes y prácticas docentes, se pueden mencionar los desarrollados por Aquino y Ferreyra (2009), Funes (2013); Gonzalez (2014a), Meschiany (2011) basados en entrevistas y Pappier (2005/2006) basado en análisis de carpetas de estudiantes.

5 Desde luego, no es la única. Otros autores, como Foucault y Marin, también han hecho interesantes aportes tal como expone Roger Chartier (1996). Desde la sociología, Bourdieu (1997) y más recientemente las relecturas de Lahire $(2005,2006,2008)$ también aportan elementos para pensar las prácticas.

6 Para este trabajo se han analizado 26 carpetas de distintos cursos de secundaria producidas entre los años 2009 y 2014, perteneciendo 12 a estudiantes de escuelas públicas y 14 de escuelas privadas. Las carpetas son el dispositivo más usual para el trabajo de los alumnos en secundaria. A diferencia de los cuadernos (utilizados en primaria), se caracterizan por reunir un conjunto de hojas que no están ligadas o pegadas a un mismo soporte sino sostenidas por argollas, por lo que pueden incluirse, mezclarse y quitarse. En relación con las carpetas como fuentes de investigación, y teniendo en cuenta sus límites y potencialidades, se toma en cuenta lo apuntado por Chartier (2002), Gvirtz (1999) y Finocchio (2005) que han estudiado cuadernos escolares. Sobre las observaciones escolares y de clases, resultan interesantes los aportes de Rockwell (1997 y 2009).

7 La escuela secundaria obligatoria en Provincia de Buenos Aires se organiza en seis años. Los tres primeros corresponden a la Educación Secundaria Básica 
La Historia Enseñada

(edades de 12-13 años a 14-15 años) y los terceros últimos a la Educación Secundaria Superior (edades 15-16 años a 17-18 años) con distintas orientaciones (Ciencias Naturales, Ciencias Sociales, Economía, Comunicación, Artes, Educación física y Lenguas extranjeras). Historia está presente como disciplina común en todos los años de la secundaria básica (en primer año integrando el área de ciencias sociales). En el caso de la secundaria superior, se incluye en todas las modalidades en $4^{\circ}$ y $5^{\circ}$ año mientras que para las modalidades de Arte y Ciencias Sociales también lo está en $6^{\circ}$ año. En relación con las clases, se han tomado observaciones realizadas entre 2012 y 2014 en nueve cursos de

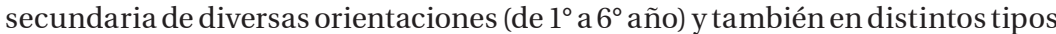
de escuelas sumando 47 horas reloj. Si bien la investigación no tiene ánimos de representatividad, la composición de la muestra -en términos de tipos de gestión de las escuelas- se correlaciona con los porcentajes de tipos de gestión escolar en partidos de las regiones educativas VI y IX donde desarrollo el trabajo de campo y la recolección de fuentes escolares. Sobre las regiones educativas, puede consultarse el mapa en: < http://servicios2.abc.gov.ar/lainstitucion/ organismos/planeamiento/imagenes/MAPA\%20REGIONES\%20A3.pdf>.

8 Como señaló Jackson (1996, p. 41), "la vida en las aulas resulta un asunto demasiado complejo como para ser observado o considerado desde una sola perspectiva". En este sentido, cabe señalar que las fuentes ponderadas en este trabajo forman parte de un corpus mayor de la investigación que también incluye siete libros de textos escolares de diversas editoriales publicados entre 2010 y 2013, un conjunto de diez revistas de consejos pedagógicos para profesores publicadas por la editorial EDIBA entre 2008 y 2010, diez planificaciones docentes (cinco de profesores observados y cinco de profesores no observados), diseños curriculares y legislación educativa (tanto la vigente como la aprobada desde 1993), etcétera. Además, mantuve entrevistas con a 5 de los profesores observados y charlas antes y después de las clases observadas con todos los profesores. De todo este corpus, que me aportó indicios para un marco interpretativo mayor, se señalarán algunos indicios secundarios a lo largo de este escrito.

9 La fragmentación, diversidad y desigualdad del mundo escolar en Argentina ha sido documentada y analizada por diversas investigaciones, por ejemplo la coordinada por Tiramonti (2004). Esta autora indica que lo que antes se llamaba sistema educativo actualmente no es más que un agregado institucional fragmentado donde es difícil reconocer sentidos compartidos. Lo que se registra es una explosión de sentidos, cada uno de los cuales se construye en el diálogo entre las expectativas familiares, las estrategias sociales de los distintos grupos y los recursos institucionales. La autora agrega que tal heterogeneidad no se corresponde linealmente con el estrato socioeconómico al que pertenecen los alumnos, sino, sobre todo, con la lectura que las familias y las instituciones realizan de las oportunidades que el contexto cambiante ofrece a sus hijos y alumnos. Así, se registran escuelas de elite (que apuntan a la preservación de tradiciones o la conformación de personalidades competitivas); las escuelas que atienden a las clases medias (que incluyen aquellas que estimulan la creatividad personal así como las que ofrecen anclar en un mundo complejo) y finalmente las escuelas que buscan generar un espacio para habitar el derrumbe, proporcionando asistencia material y pedagógica.

10 Esta caracterización no surge de investigaciones sistemáticas de la historia de la enseñanza de la historia puesto que son pocos los estudios sobre las prácticas en la enseñanza de la historia en la primera mitad del siglo XX, siendo una excepción la pesquisa de Liliana Aguiar para el caso del colegio Montserrat de

764 Educação \& Realidade, Porto Alegre, v. 42, n. 2, p. 747-769, abr./jun. 2017. 
Córdoba, dependiente de la Universidad de la provincia homónima (2010). No obstante, se puede reconstruir el funcionamiento de esta disciplina a partir de otros indicios surgidos de estudios sobre las prácticas en las aulas antes de la reforma educativa de los '90 (Finocchio; Lanza, 1993); indagaciones sobre los saberes en la enseñanza de la historia en el siglo XX plasmados en libros de textos escolares (Finocchio, 1991; 1999; Romero, 2004; De Amézola, 2008); investigaciones sobre las prácticas en la historia escolar realizada en otros países - por caso, el estudio de Cuesta (1997) en España -; fuentes de la primera mitad del siglo XX (informes de inspectores, sugerencias de actividades en revistas educativas, orientaciones para la realización de ejercicios en las aulas de planes y programas); publicaciones didácticas y pedagógicas así como materiales para la innovación editados en momentos de la reforma educativa de los '90 que nos permiten inferir las prácticas que se propusieron cambiar.

11 Por razones de espacio, utilizaré extractos de transcripciones de observaciones de clases y de carpetas de estudiantes. No obstante, tomaré también en cuenta en el análisis los datos arrojados por otras fuentes escolares y docentes que se han enumerado en la nota 8.

12 En la normativa, la exposición del docente casi no es mencionada y cuando es citada se la señala como una tarea secundaria, posterior y ordenadora del trabajo de los estudiantes "[...] el docente podrá realizar una exposición clara y concisa para ordenar las ideas y procesos que se suceden en diferentes escalas y que se han mostrado deshilvanados durante el trabajo" (Orientaciones didácticas del Diseño Curricular para $3^{\circ}$ año (ES), Ciencias Sociales, p. 46). En las obras didácticas -por ejemplo, Quinquer (1997) - se pone énfasis en las estrategias de trabajo cooperativo por parte de los estudiantes por sobre la exposición docente.

13 Véase, por ejemplo, Aisemberg (1995).

14 Para la reproducción de las transcripciones de clases observadas se han utilizado algunas convenciones tales como: XXX para fragmentos inaudibles, [...] para marcar el recorte de la transcripción,$==$ para señalar la simultaneidad en el habla, las itálicas para señalar el énfasis en el habla, el signo _ para evidenciar autorreparaciones en el discurso. Entre paréntesis se colocan algunas aclaraciones o se describen actos no verbales. Las negritas han sido utilizadas a los efectos de ponderar algunos detalles para este trabajo.

15 Esto también aparece en otras fuentes tales como revistas para docentes y libros de textos escolares. Por ejemplo: "Respondé y debatí con tus compañeros: ¿Qué opinión les merece la actitud de Alfonsín de retirarse del gobierno? ¿Qué habrá privilegiado en ese momento? ¿Por qué?” (Revista de la Educación Secundaria, EDIBA, n. 120, p. 17, 2009). "El escritor uruguayo Eduardo Galeano dice que las líneas férreas son las "venas abiertas de América Latina", frase que le da nombre a uno de sus libros más famosos. ¿Por qué consideras que dice eso? Escribí tu argumento y debatilo con tus compañeros de clase (Revista de la Educación Secundaria, EDIBA, n. 119, Cuadernillo, p. 13, 2009). “¿Cuál es tu opinión respecto del conflicto de las exportaciones del campo que hemos vivido desde hace un tiempo en el país?" (Revista de la Educación Secundaria, EDIBA, n. 119, Cuadernillo, p. 13, 2009 ).

16 Varias orientaciones didácticas editadas en momentos de la reforma educativa de la Ley Federal de 1993, señalaban la utilidad de las redes conceptuales. Véase, por ejemplo, Cuadernillo Nueva escuela, n. 21, Historia, p. 45.

17 Los profesores que utilizan estas tareas manifestaron en las entrevistas con que son actividades realizadas porque son saberes que consideran claves 
La Historia Enseñada

para los estudiantes, para que "aprendan a aprender", "aprendan a estudiar", y "estudien para seguir estudiando". De alguna manera, esto se relaciona con las finalidades actuales de la secundaria obligatoria que tiene como finalidad principal "habilitar a los/las adolescentes y jóvenes para el ejercicio pleno de la ciudadanía, para el trabajo y para la continuación de estudios". Al respecto, véase, Ley de Educación Nacional 26206/06, artículo 30 (Argentina, 2006).

18 Esto último se repite sobre todo en las aulas donde los estudiantes no usan libros escolares y los profesores manifiestan (en las entrevistas) que dictan esas recapitulaciones buscando que "les quede algo en la carpeta para que estudien desde allí".

19 De las entrevistas y charlas mantenidas con los profesores se desprende que las láminas se realizan por, al menos, dos razones. Por un lado, porque ayudan a los estudiantes a aprender a realizar una síntesis y a exponer lo trabajado y, por otro, porque cumplen el rol de hacer más ameno el lugar de trabajo.

20 Sobre esta cuestión, véase la nota 9.

21 Las itálicas son mías.

\section{Referencias}

AGUIAR, Liliana. Apuntes para una historia de la disciplina escolar historia en nuestro territorio. El período fundante, desde una mirada del interior del país. In: ROITENBURD, Silvia; ABRATTE, Juan Pablo (Comp.). Historia de la Educación Argentina: del discurso fundante a los imaginarios reformistas contemporáneos. Córdoba: Editorial Brujas, 2010. P. 26-42.

ARGENTINA. Ley no 26.206 de 14 de diciembre de 2006. Ley de Educación Nacional. Artículo 30. Argentina, 27 dic. 2006. Disponible en: <http://www.me.gov. ar/doc_pdf/ley_de_educ_nac.pdf $>$. Aceso en: 26 nov. 2016.

AQUINO, Nancy; FERREYRA, Susana. Los "textos vivos" y la enseñanza de la historia en Córdoba. In: JORNADAS INTERESCUELAS/ DEPARTAMENTOS DE HISTORIA, XII, 2009; Bariloche. Actas... Bariloche, 2009.

AISEMBERG, Beatriz. Para qué y cómo trabajar con los conocimientos previos de los alumnos. In: AISEMBERG, Beatriz; ALDEROQUI, Silvia. Didáctica de las Ciencias Sociales. Aportes y reflexiones. Buenos Aires: Paidós, 1995. P. 137-162.

AISEMBERG, Beatriz. La lectura en la enseñanza de la historia: las consignas del docente y el trabajo intelectual de los alumnos. Lectura y Vida, Buenos Aires, año 26, n. 3, p. 22-31, 2005.

BOURDIEU, Pierre. El sentido Práctico. Buenos Aires: Siglo XXI, 1997.

CHARTIER, Anne Marie. Um dispositivo sem autor: cadernos e fichários na escola primária. Revista Brasileira de História da Educação, São Paulo, v. 2. n. 1, p. 9-26, ene./jun. 2002.

CHARTIER, Anne Marie. Fazeres ordinários da classe: una aposta para a pesquisa e para a formação. Educação e Pesquisa, São Paulo, v. 26, n. 2, p. 57-168, jul./dic. 2000.

CHARTIER, Roger. Escribir las Prácticas. Foucault, De Certeau, Marin. Buenos Aires: Manantial, 1996.

CHERVEL, André. L'école, lieu de production d'une culture. In: AUDIGIER, François; BAILLAT, Gilles (Dir.). Didactique de l'Histoire, de la Géographie, des Sciences Sociales. Analyser et gérer les situations d'enseignement-apprentissage. Paris: INRP, 1992. P. 195-198.

766 Educação \& Realidade, Porto Alegre, v. 42, n. 2, p. 747-769, abr./jun. 2017. 
CHERVEL, André. Historia de las disciplinas escolares: reflexiones sobre un campo de investigación. Revista de Educación, Madrid, n. 295, p. 59-111, 1991. CUESTA, Raimundo. Sociogénesis de una Disciplina Escolar: la historia. Barcelona: Pomares-Corredor, 1997.

DE AMÉZOLA, Gonzalo. Esquizohistoria. La historia que se enseña en la escuela, la que interesa a los historiadores y un cambio posible de la historia escolar. Buenos Aires: Libros del Zorzal, 2008.

DE CERTEAU, Michel. La Invención de lo Cotidiano. Artes de hacer. México: Universidad Iberoamericana, 2007.

FELDMAN, Daniel. Imágenes en la historia de la enseñanza: la lámina escolar. Educação \& Sociedade, Campinas, v. 25, n. 86, p. 75-101, abr. 2004.

FINOCCHIO, Silvia. Programas y textos en la historia de cuatro asignaturas de la escuela media: Historia, Lengua y Literatura, Educación Cívica y Física. Propuesta Educativa, Buenos Aires, año 1, n. 1, p. 51- 63, 1989.

FINOCCHIO, Silvia. ¿Qué llega de nuestra investigación a la escuela media? Una reflexión para los historiadores. Entrepasados, Revista de Historia, Buenos Aires, v. 1, n. 1, p. 93-106, 1991.

FINOCCHIO, Silvia. Cambios en la enseñanza de la historia: la transformación argentina. Iber, Didáctica de las Ciencias Sociales, Geografía e Historia, Barcelona, v. 22, n. 1, p. 17-30, 1999.

FINOCCHIO, Silvia. Apariencia escolar. In: DUSSEL, Inés; FINOCCHIO, Silvia (Comp.). Enseñar Hoy. Una introducción a la educación en tiempos de crisis. Buenos Aires: Fondo de Cultura Económica, 2003. P. 81-87.

FINOCCHIO, Silvia. La ciudadanía en los cuadernos de clase. Enseñanza de las Ciencias Sociales, Revista de Investigación, Barcelona, año IV, n. 4, p. 3-10, 2005.

FINOCCHIO, Silvia. Historia, memoria y educación en Argentina. De aprender de memoria a enseñar para la memoria la historia argentina reciente. In: PAGÈS, Joan; GONZALEZ, María Paula (Comp.). Història, Memòria i Ensenyament de la Història. Perspectives europees i llatinoamericanes. Barcelona: Servei de Publicacions UAB, 2009. P. 83-101.

FINOCCHIO, Silvia. Los docentes, los saberes y la mutación de la escuela. In: FINOCCHIO, Silvia; ROMERO, Nancy (Comp.). Saberes y Prácticas Escolares. Rosario: HomoSapiens, 2011. P. 175-199.

FINOCCHIO, Silvia; LANZA, Hilda. ¿Cómo se conforma la práctica docente? Una aproximación a los ámbitos que constituyen el discurso de los profesores de historia del nivel medio. In: LANZA, Hilda; FINOCCHIO, Silvia. Curriculum Presente, Ciencia Ausente. Tomo III. Buenos Aires: FLACSO-CIID-Miño y Dávila, 1993. P. 101-179.

FUNES, Graciela. Historias Recientes Enseñadas. Utopías y prácticas. Neuquén: EDUCO, 2013.

GONZALEZ, María Paula. Historia en el nivel secundario en Argentina hoy: notas sobre el funcionamiento de una disciplina escolar. História \& Ensino, Londrina, v. 19, n. 2, p. 07-22, jul./dic. 2013.

GONZALEZ, María Paula. La Historia Argentina Reciente en la Escuela. Saberes y prácticas docentes en torno a la última dictadura. Los Polvorines: Publicaciones UNGS, 2014a.

GONZALEZ, María Paula. Investigaciones sobre la enseñanza de la historia en Argentina. Notas para un balance de dos décadas. In: PLÀ, Sebastian; PAGÈS, 
Joan (Comp.). La Investigación en la Enseñanza de la Historia en América Latina. México: Publicaciones de la Universidad Pedagógica Nacional y Bonilla Artigas Editores, 2014b. P. 39-70.

GVIRTZ, Silvina. El Discurso Escolar a través de los Cuadernos de Clase: Argentina 1930-1970. Buenos Aires: Eudeba, 1999.

JACKSON, Philip. La Vida en las Aulas. Madrid: Morata, 1996.

JULIA, Dominique. A cultura escolar como objeto histórico. Revista Brasileira de História da Educação, São Paulo, v. 1, n. 1, p. 9-43, ene./jun. 2001.

LAHIRE, Bernard. Pensar la acción: entre la pluralidad disposicional y la pluralidad de contextos. In: TIRAMONTI, Guillermina; MONTES, Nancy (Comp.). La Educación Media a Debate. Buenos Aires: Manantial- FLACSO, 2008. P. 13-21.

LAHIRE, Bernard. Lógicas prácticas: el "hacer" y el "decir sobre el hacer". In: LAHIRE, Bernard. El Espíritu Sociológico. Buenos Aires: Manantial, 2006. P. 137-155.

LAHIRE, Bernard. De la teoría del habitus a una sociología psicológica. In: LAHIRE, Bernard (Dir.). El Trabajo Sociológico de Pierre Bourdieu. Deudas y críticas. Buenos Aires: Siglo XXI, 2005. P. 143-179.

MARONNA, Mónica; SÁNCHEZ VILELA, Rosário. La puesta en relato de lo cotidiano. In: RICO DE SOTELO, Carmen (Coord.). Relecturas de Michel de Certeau. México: Universidad Iberoamericana, 2006. P. 93-126.

MESCHIANY, Talia. Relato de una investigación: enseñanza de la historia y educación para la ciudadanía en el contexto de la crisis del año 2001. Clío y Asociados. La historia enseñada, La Plata, n. 15, p. 53-76, 2011.

MONTEIRO, Ana María. Professores de História. Entre saberes e práticas. Rio de Janeiro: MauadX, 2007.

MONTEIRO, Ana María. Entre o estranho e o familiar: o uso de analogias no ensino de história. Cadernos CEDES, Campinas, v. 25, n. 67, p. 333-347, dic. 2005.

MONTEIRO, Ana María; PENNA, Fernando. Ensino de História: saberes em lugar de fronteira. Educação e Realidade, Porto Alegre, v. 36, n. 1, p. 191-211, ene./abr. 2011. MONTEIRO, Ana María; GASPARELLO, Arlette; MAGALHÃES, Marcelo (Org.). Ensino de História. Sujeitos, saberes e práticas. Rio de Janeiro: MauadX FAPERJ, 2004

PAPPIER, Viviana. Reescritura de la Historia en el aula luego de la Reforma Educativa. Una mirada a las carpetas de Ciencias Sociales de $7 \mathrm{mo}$ año. Clío y Asociados. La historia enseñada, La Plata, v. 9-10, p. 84-102, 2005-2006.

QUINQUER, Dolors. Estrategias de enseñanza: los métodos interactivos. In: BENEJAM, Pilar; PAGÉS, Joan (Coord.). Enseñar y aprender Ciencias Sociales, Geografía e Historia en la Educación Secundaria. Barcelona: Horsori, 1997. P. 97-121. ROCKWELL, Elsie (Coord.). La Escuela Cotidiana. Buenos Aires: FCE, 1997.

ROCKWELL, Elsie. La Experiencia Etnográfica. Historia y cultura en los procesos educativos. Buenos Aires: Paidós, 2009.

ROMERO, Luis Alberto (Coord.). La Argentina en la Escuela. La idea de nación en los textos escolares. Buenos Aires: Siglo XXI, 2004.

TIRAMONTI, Guillermina (Comp.). La Trama de la Desigualdad Educativa. Mutaciones recientes en la escuela media. Buenos Aires: Manantial, 2004.

TORRES SANTOMÉ, Jurjo. La práctica reflexiva y la comprensión de lo que acontece en las aulas. Prólogo a la edición castellana. In: JACKSON, Philip. La Vida en las Aulas. Madrid: Morata, 1996. P. 11-24.

768 Educação \& Realidade, Porto Alegre, v. 42, n. 2, p. 747-769, abr./jun. 2017. 
TYACK, David; CUBAN, Larry. En Busca de la Utopía. Un siglo de reformas de las escuelas públicas. Buenos Aires: Fondo de Cultura Económica, 2001.

VIÑAO, Antonio. Sistemas Educativos, Culturas Escolares y Reformas: continuidades y cambios. Madrid: Morata, 2002.

VINCENT, Guy, LAHIRE, Bernard ; THIN, Daniel. Sur l'histoire et la théorie de la forme scolaire. In: VINCENT, Guy (Dir.) L'Éducation Prisonnière de la Forme Scolaire? Scolarisation el socialisation dans les sociétés industrielles. Lyon: Presses Universitaires de Lyon, 1994. p. 11-48.

Maria Paula Gonzalez és graduada en Historia por la Universidad de Buenos Aires, Argentina y Doctora por la Universidad Autónoma de Barcelona en el programa de Didáctica de las Ciencias Sociales. Actualmente se desempeña como docente de grado y posgrado en el Instituto del Desarrollo Humano de la UNGS y como investigadora de carrera del CONICET.

E-mail: gonzalezamorena@gmail.com 